\title{
UNO STRANO TRAPIANTO TRA DARWIN E MANTEGAZZA
}

\author{
CARLA GARBARINO (*), PAOLO MAZZARELLO (**)
}

Nota presentata dal m.e. Paolo Mazzarello

(Adunanza del 27 giugno 2013)

SunTO. - Durante l'allestimento, curato dall'Università di Pavia, di una mostra organizzata nel centenario della morte di Paolo Mantegazza (1910), nel Museo per la Storia dell'Università e stato trovato uno strano corno cheratinico, accompagnato da un cartellino che lo identificava come "innesto di sperone di gallo sull'orecchio di una vacca". Dopo alcune ricerche storiche abbiamo scoperto che questo strano oggetto fu al centro di una corrispondenza scientifica tra Mantegazza e Charles Darwin, che fece riferimento al preparato nel suo libro The Variation of Animals and Plants under Domestication.

$$
* * *
$$

AвSTRACT. - In the centennial anniversary of the death of the Italian pathologist, anthropologist and writer Paolo Mantegazza (1910), the University of Pavia planned a commemorative exhibition to show the versatile aspects of his scientific and literary activity. In the course of the research to prepare the exposition, a strange cheratinic horn was found at the Museum for the History of the University of Pavia labeled as 'spur of a cock transplanted into an ear of a cow'. At the end of some historical investigation, we found that this strange object was at the centre of a scientific correspondence between Mantegazza and Charles Darwin, who made reference to it in his book The Variation of Animals and Plants under Domestication.

(*) Museo per la Storia dell’Università di Pavia, Italy.

E-mail: mariacarla.garbarino@unipv.it

(*iv) Dipartimento di Scienze del Sistema Nervoso e del Comportamento, Univer-

sità di Pavia, Italy.

E-mail: paolo.mazzarello@unipv.it 


\section{UNO STRANO PREPARATO}

Nella primavera del 1929 l'Italia fascista celebrava con orgoglio le straordinarie scoperte scientifiche in cui si riverberava il genio della nazione con una grande mostra organizzata a Firenze, nel Palazzo delle Esposizioni ([1], pagg. 46-48). Si trattava di un importante evento, destinato a riscuotere un grande successo di pubblico, con circa diecimila oggetti disposti in una narrazione espositiva suddivisa per aree geografiche. Al primo piano due sale erano dedicate alla Lombardia, comprendendo Milano, Pavia, Bergamo e Brescia. L'Università di Pavia aveva contribuito inviando alcuni cimeli - selezionati da una commissione presieduta da Achille Monti ${ }^{1}$ - legati all'attività di Alessandro Volta, Lazzaro Spallanzani, Antonio Scarpa, Bartolomeo Panizza, Camillo Golgi e di altri scienziati che avevano reso famoso l'Ateneo ticinese. Tra questi cimeli si trovava un preparato, scelto come testimonianza dei primi significativi esperimenti di trapianto interspecie: un corno ricurvo, montato su un piedestallo di legno nero con un piccolo cartellino di accompagnamento che recitava "innesto di sperone di gallo sull'orecchio di una vacca" (Fig. 1). Il pezzo era appartenuto a Paolo Mantegazza, un personaggio ben noto nella città dell'esposizione, essendo stato titolare, proprio a Firenze, della cattedra di Antropologia e fondatore del Museo di Antropologia ed Etnologia.

Sei mesi più tardi, alla conclusione della mostra, il pezzo fu restituito a Pavia, entrando poi a far parte del neo-istituito Museo per la Storia dell'Università, dove rimase seminascosto e quasi dimenticato su uno scaffale per circa ottanta anni, fino a che, nel 2010, siamo stati in grado di ricostruirne l'affascinante storia, legata al nome di Charles Darwin.

\section{Gli innesti animali di PaOlo MantegazZA}

Nel 1865 Paolo Mantegazza (1831-1910), a quel tempo professore di Patologia generale all'Università di Pavia, pubblicò un lavoro dal titolo Degli innesti animali e della produzione artificiale delle cellule,[2] in cui descriveva uno strano preparato anatomico:

1 Documenti relativi alla sezione pavese dell'Esposizione si conservano nell'archivio del Museo per la Storia dell'Università; altre notizie sono rintracciabili nei verbali del Consiglio di Amministrazione dell'Università di Pavia. Una mole rilevante di documenti è anche conservata nell'archivio del Museo Galileo di Firenze. 
Nel Brasile venne innestato lo sperone di un gallo nell'orecchio d'una vacca, dove trovò terreno per attecchire e crescere indefinitamente. Vi rimase otto anni, crescendo sempre fino a simulare un terzo corno, e distaccato poi come curiosità mi fu gentilmente donato dal mio ottimo collega prof. Balsamo Crivelli. ${ }^{2}$

Questo sperone pesa 396 grammi, ed ha un'altezza di 24 centimetri. La sua curva maggiore ha uno sviluppo di 52 centimetri e la minore è di 25 centimetri. La base ha contorni irregolari ... ed ha una circonferenza di $20 \mathrm{~cm}$. (Fig. 2).

Questo fatto interessante per la sua rarità e più ancora per lo straordinario sviluppo dell'organo trapiantato ci presenta un tipo parassitico, in cui il tessuto innestato, senza misura e senza legge di coordinazione organica cresce, sempre a spesa dell'organismo in cui è portato ... ([2], pag. 51).

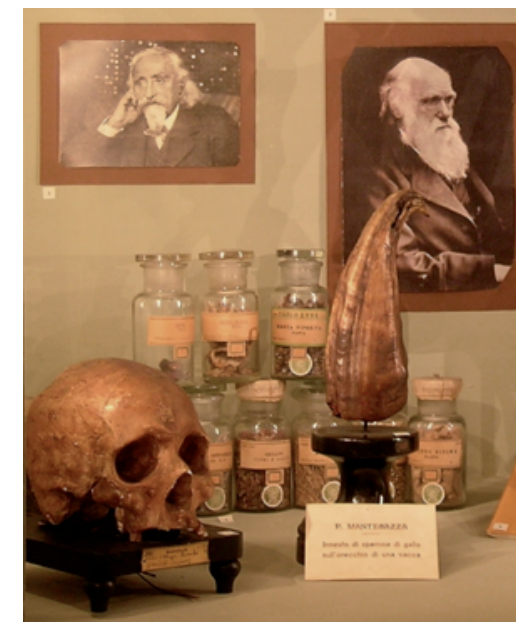

Fig. 1 - La vetrina del Museo per la Storia dell'Università in cui è esposto il preparato.

Il testo era accompagnato da un disegno esplicativo, eseguito da un allievo di Mantegazza, lo studente di medicina Giulio Bizzozero (1846$1901)^{3}$ che si era guadagnato la stima del suo professore dimostrando una grande abilità nella ricerca scientifica (Fig. 3).

2 Giuseppe Balsamo Crivelli (1800-1874) docente di Zoologia e Anatomia comparata all'Università di Pavia.

3 Bizzozero divenne professore di Patologia generale all'Università di Pavia e successivamente a Torino.[3] 


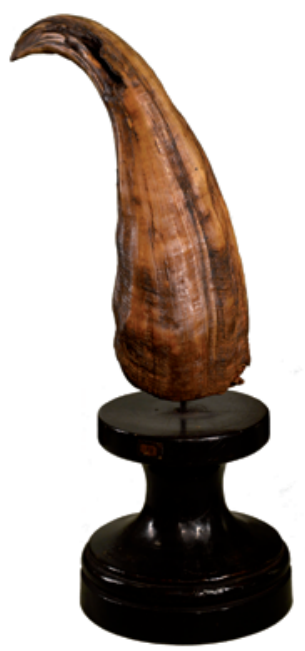

Fig. 2 - Il preparato etichettato come "Innesto di sperone di gallo sull'orecchio di una vacca". Sistema Museale di Ateneo - Museo per la Storia dell'Università di Pavia.

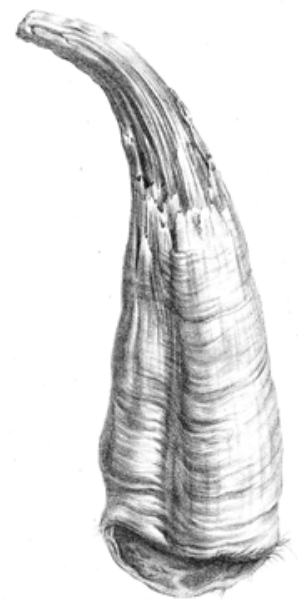

Fig. 3 - Il disegno realizzato da Giulio Bizzozero dello sperone di gallo trapiantato sull'orecchio di una vacca pubblicato nel volume di Paolo Mantegazza, Degli innesti animali e della produzione artificiale delle cellule, Milano, Amministrazione del Politecnico, 1865, Tav. 3. 
Nato a Monza, Paolo Mantegazza aveva studiato medicina all'Università di Pavia, laureandosi nel 1854. Sei anni dopo aveva ottenuto la cattedra di Patologia generale, tornando nella città dei suoi studi dopo un lungo periodo trascorso viaggiando attraverso il sud America, in cerca di fortuna e di esperienza, soprattutto in campo scientifico e medico. Mantegazza era uno scienziato eclettico, destinato a diventare uno dei più noti intellettuali italiani della seconda metà del XIX secolo. Scrittore prolifico, con un'ampia varietà di interessi, si occupò di patologia, fisiologia, farmacologia, antropologia, sessuologia, etnologia ma fu anche un politico e un deputato, un novellista famoso e un avventuroso viaggiatore.[4] (Fig. 4).

La sua curiosità intellettuale lo spinse ad approfondire diversi argomenti della medicina contemporanea, fra i quali il problema dei trapianti. Per perseguire i suoi scopi allestì, nel 1862, un piccolo laboratorio di patologia sperimentale nel quale, tra le difficoltà causate dalla scarsità dell'equipaggiamento (lo scienziato fu costretto a portare da casa microscopi e strumenti di sua proprietà) e dalla mancanza di riscaldamento dei locali, condusse le sue ricerche con l'aiuto di alcuni brillanti studenti di medicina.

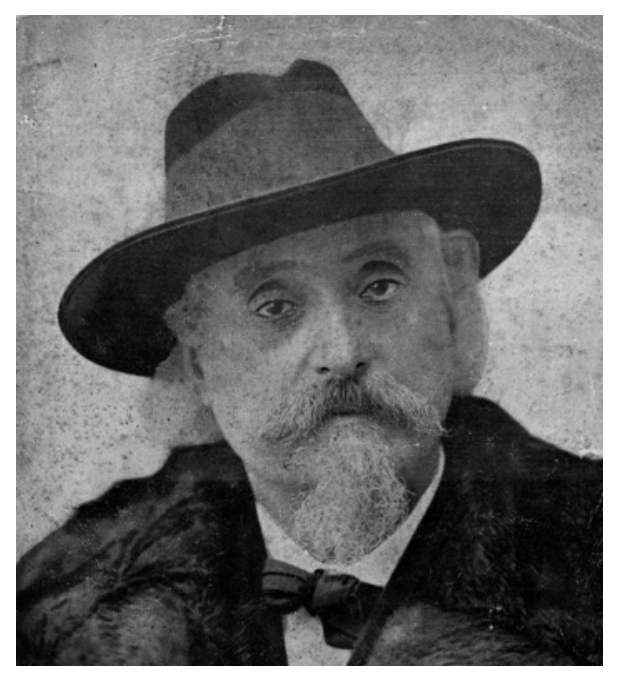

Fig. 4 - Paolo Mantegazza. Sistema Museale di Ateneo - Museo per la Storia dell'Università di Pavia. 
L'opera Degli innesti animali raccoglieva i risultati di centinaia di esperimenti condotti su diverse specie animali (come rane, topi, cani e cavie). Mantegazza scoprì che i due elementi portati in contatto (il tessuto prelevato e il corpo in cui esso era stato innestato) esercitavano l'uno sull'altro un'influenza reciproca, che dipendeva da diversi fattori come la massa del tessuto innestato, la specie da cui esso era stato prelevato e quella su cui era stato trapiantato, la salute e la robustezza dell'animale ricettore. Qualche volta il tessuto innestato si decomponeva ed era possibile osservare un processo di suppurazione, reazioni che si potevano riassumere con il tentativo dell'organismo di rimuovere un corpo estraneo. Talvolta, tuttavia, l'influenza reciproca era più complessa e il tessuto innestato, trovando nella nuova sede alcune condizioni necessarie alla sua esistenza, continuava a vivere nutrendosi "a spese dell'organismo in cui fu portato e diviene un vero membro di esso o un tumore patologico che ne fa parte" ([2], pag. 52). Secondo Mantegazza i tessuti e gli organi avevano "una vita autonoma assai più indipendente e più gagliarda" di quanto si potesse immaginare prima dello sviluppo della teoria cellulare e della patologia cellulare del medico tedesco Rudolf Virchow (1821-1902) ([2], pag. 53). Gli esperimenti di innesti animali confermavano pienamente "la vita indipendente delle cellule" ([2], pag. 53) anche se, in qualche modo contraddittoriamente, Mantegazza non accettò l'aforisma di Virchow omnis cellula ex cellula ipotizzando invece, in particolari condizioni, una sorta di generazione spontanea.[5]

Mantegazza era convinto che quelle ricerche sui trapianti avrebbero fornito materiale prezioso alla fisiologia e all'istologia e le premesse per lo sviluppo di nuove tecniche chirurgiche.

Il lavoro sugli innesti animali venne brevemente riassunto in una nota editoriale dal titolo "The Results of Engrafting Animal Tissues" pubblicata sulla rivista The Popular Science Review:

A remarkable memoir, detailing the results of some curious experiments regarding the vitality of engrafted animal tissues, has been written by Signor Mantegazza. He has engrafted the organs of one animal upon the body of another with the most surprising consequences. Some of the tissues thus engrafted underwent fatty degeneration; but others appeared to live as well in the new as in the old organism, being united in course of time to the new one by bloodvessels and connective tissues... The spleen can exist for a long while in the body of another animal, and may even increase in weight. The spur of a cock lived for a period of eight years in the eye of an ox, and acquired a weight of 396 grams. [6] 
Il Popular Science Review era diffuso in tutto il mondo anglofono presso un vasto pubblico di lettori. Charles Darwin era tra questi.

\section{DARWIN E IL PROBLEMA DELL'EREDITARIETÀ: \\ LA TEORIA DELLA PANGENESI}

Nel 1865 Charles Darwin era alle prese col problema dell'ereditarietà. La trasmissione dei caratteri ereditari lo aveva sempre messo in difficoltà poiché nella sua teoria dell'evoluzione l'origine delle variazioni ereditarie sulle quali agiva la selezione naturale rimaneva misteriosa.

Tuttavia Darwin aveva alla fine messo a punto una teoria provvisoria, chiamata pangenesi, che gli permetteva di spiegare le differenze nella prole e le ragioni della loro ereditarietà ([7], pag. 275). Si trattava di un'ipotesi strumentale a supporto della teoria dell'evoluzione per rispondere alle critiche mosse a On the Origin of Species.

Per dar conto delle differenze e somiglianze dei membri di una famiglia, Darwin ipotizzò che i diversi tessuti del corpo godessero di una relativa indipendenza gli uni dagli altri e che all'interno di questi si trovassero alcune particelle biologiche, chiamate 'gemmule'. Secondo Darwin queste gemmule avevano la capacità di generare una parte dell'organismo simile a quella da cui erano derivate. Erano considerate specifici pacchetti di informazioni, ciascuno dei quali era capace di tenere memoria dei tessuti originali anche se non dell'intero organismo ([7], pag. 276). In altre parole queste particelle avevano la proprietà di innescare la struttura tridimensionale di quella parte del corpo dalla quale si erano originate. Quindi gemmule provenienti da un braccio potevano generare un braccio, gemmule provenienti da un occhio, un occhio e così via. Queste particelle, secondo Darwin, si moltiplicavano e potevano raggiungere le gonadi, dalle quali erano poi trasmesse dai genitori alla prole. La mescolanza delle gemmule rendeva conto della mescolanza delle caratteristiche della discendenza che riceveva gruppi di esse in parte dalla madre e in parte dal padre. Alcune di esse potevano anche rimanere nascoste attraverso le generazioni per poi manifestarsi improvvisamente ([7], pag. 276).

Darwin era ansioso di trovare evidenza della relativa indipendenza delle gemmule che componevano i tessuti, un aspetto che egli considerava un indiretto supporto in favore della sua teoria della pangenesi. Mentre raccoglieva materiale sull'ereditarietà e faceva ricerche su piante e animali, si imbatté nel sunto del lavoro di Mantegazza pubblicato 
sul Popular Science Review. Fu probabilmente colpito dallo strano corno che era cresciuto dopo l'innesto in un altro tessuto di una specie differente. Le gemmule contenute nello sperone trapiantato avevano forse ripreso a crescere indipendentemente nella nuova sede?

Le idee sull'ereditarietà sviluppate da Darwin divennero il cuore concettuale della sua nuova opera in due volumi, The Variation of Animals and Plants under Domestication, pubblicata nel gennaio 1868. Nel secondo volume Darwin citò, accanto a risultati simili ottenuti da altri ricercatori, anche una parte della recensione del lavoro di Mantegazza del Popular Science Review:

Many facts support this view of the independent life of each minute element of the body. Virchow insists that a single bone-corpuscle or a single cell in the skin may become diseased. The spur of a cock, after being inserted into the eye of an ox, lived for eight years, and acquired a weight of 306 grammes, or nearly fourteen ounces ([8], pag. $\left.369^{4}\right)$.

C'erano due errori in questa citazione: il primo riguardava il sito dell'innesto (lo sperone era stato trapiantato nell'orecchio e non nell'occhio) ed era già presente nel Popular Science Review; il secondo, che riguardava il peso del pezzo innestato, era stato commesso da Darwin stesso.

\section{IL CONTATTO TRA I DUE SCIENZIATI}

Mantegazza, che era un appassionato ammiratore di Darwin, fu elettrizzato quando vide il suo nome citato nel The Variation e subito scrisse allo scienziato inglese. È assai probabile che la ragione dichiarata dalla lettera - correggere questi due errori - non fosse niente di più che un semplice pretesto per entrare in relazione con il creatore della teoria dell'evoluzione.

In una lettera datata da Pavia, il 19 marzo 1868, dopo essersi complimentato con Darwin per la sua "grand ouvrage", The Variation of Animals and Plants under Domestication, "un monument sublime de

4 La nota 22 del capitolo XXVII recita "Mantegazza, quoted in 'Popular Science Review', July 1865, p. 522”. 
l'intelligence humaine", aver benedetto lo scienziato inglese "au nom de la science" e "au nom des admirateurs de la nature", Mantegazza passava a fare le due piccole precisazioni per rettificare le inesattezze, certamente imputabili al Popular Science Review, promettendo di inviare copia dell'opuscolo originale: ${ }^{5}$

Dans le second volume (pag. 369) j'ai eu le plaisir de voir cité un de mes travaux sur la greffe animale; mais vous vous êtes rapporté à le Popolar Science Review, où on a decrit nous experience avec peu d'exactitude. L'ergot du coq n'a pas été greffé dans l'oeil d'un beuf, mais dans une oreille (cela se fait souvent dans le Bresil); et après huit ans il ne pesait pas 306, mais 396 grammes. Je vous envoie mon ouvrage original sur la greffe; vous trouverez dessiné l'ergot à la table $3^{a}$ et décrit à pag. 51.

Mantegazza includeva poi nella lettera anche un estratto dal suo più recente lavoro sui matrimoni tra consanguinei,[9] promettendo di mandare di più non appena l'opera fosse stata pubblicata.[10] ${ }^{6}$ Prima di firmarsi come "Votre admirateur $\mathrm{D}^{\mathrm{r}}$. Paul Mantegazza" si affrettava a spiegare che gli avrebbe inviato il proprio ritratto in segno di devozione, con il desiderio di entrare "au moins en ombre ... dans votre sanctuaire où vous reformez la science, où vous ouvrez des horizons sans bornes à la meditation et à la philosophie de l'avenir". Ci teneva poi a fargli sapere che sarebbe stato il più onorato degli uomini se Darwin gli avesse ricambiato il favore. ${ }^{7}$

Mantegazza apprezzava la teoria della pangenesi. Prima della pubblicazione del The Variation di Darwin, mentre esaminava le conseguenze dei matrimoni tra consanguinei, aveva infatti ipotizzato una sorta di modello 'corpuscolare' della trasmissione ereditaria dei caratteri biologici, sotto certi aspetti simile a quello della teoria di Darwin.[9]

5 Copia di questo lavoro non è stata trovata nella biblioteca di Darwin (vedi l'edizione on line di questa lettera, nota f4, all'indirizzo http://www.darwinproject.ac.ok), ma Darwin lo cita nella seconda edizione del The Variation (1875), 2, pag. 365.

6 Copie dell'estratto e il lavoro completo si trovano nella collezione di pamphlet di Darwin nella Biblioteca dell'Università di Cambridge. Vedi http://www.darwinproject.ac.uk, ultimo accesso 01/10/2014.

7 La lettera originale, in francese, e una traduzione inglese della stessa sono disponibili all'indirizzo http://www.darwinproject.ac.uk, ultimo accesso 01/10/2014. 
Due mesi dopo questa lettera, Mantegazza rese pubblico il suo entusiasmo per il lavoro dello scienziato inglese in una lunga recensione del The Variation nell'importante rivista Nuova Antologia.[11] Apprendiamo da questo scritto che Darwin aveva immediatamente risposto alla lettera di Mantegazza, esprimendo il suo dubbio che lo scienziato italiano non avrebbe forse approvato la teoria della pangenesi. ${ }^{8} \mathrm{Al}$ contrario, Mantegazza era dell'opinione che la teoria della pangenesi fosse "l'opera di un genio, [...] una sintesi arditissima di fatti... fin qui rimasti isolati ed incompresi", il genio di Darwin era "una delle più splendide fiaccole che abbiano illustrati in questo secolo la più oscura delle scienze" ([11], pag. 98).

Tracce della risposta di Darwin si trovano anche nel diario di Mantegazza, una monumentale registrazione della sua vita quotidiana in oltre 60 volumi che lo scienziato portò avanti dal 1848 all'anno della morte:

Da Darwin ebbi poi ringraziamenti infiniti. Mi scrive di rallegrarsi ch'io avessi pensato prima di lui alla pangenesi, e mi disse di aver mandato a Canestrini' che sta facendo la traduzione italiana un estratto del mio articolo per quell'edizione. ${ }^{10}$

\section{LA RICOSTRUZIONE DELLA STORIA}

Come le gemmule della pangenesi di Darwin, che potevano rivelarsi dopo generazioni, lo strano corno cresciuto nell'orecchio di una vacca scomparve per molti anni dopo il suo primo momento di celebrità. Poi, nel 1929, fu presentato a Firenze nella prima Esposizione Nazionale di

8 "io temo che voi non disapproviate il capitolo sulla pangenesi, ma io ho fiducia che qualche cosa di molto analogo a questa teoria sarà un giorno adottato, e questa è già l'opinione di parecchi buoni giudici in Inghilterra", ([11], pag. 97). La lettera originale di Darwin non è ancora stata trovata nell'archivio di Mantegazza ([12], pag. 190).

9 Giovanni Canestrini (1835-1900), all'epoca professore di Storia naturale a Modena poi, dal 1869, professore di Zoologia, anatomia e fisiologia comparata a Padova, era il traduttore italiano del libro di Darwin. Con Luciano Salimbeni aveva già tradotto On the Origin of Species by means of Natural Selection con il titolo Sull'origine delle specie per elezione naturale (Modena, Zanichelli, 1864).

10 Biblioteca civica di Monza, Fondo Mantegazza, Giornale della mia vita o le mie confessioni, 12-21 maggio 1868. 
Storia della Scienza, come un esempio degli studi di Mantegazza sui trapianti ma, apparentemente, senza alcun riferimento a Darwin e al problema dell'ereditarietà. Dopo la mostra questo preparato scomparve di nuovo, posto in uno scaffale del Museo per la Storia dell'Università di Pavia senza che nessuno conoscesse la sua curiosa storia. Quattro anni fa, durante l'organizzazione di una mostra in occasione del centenario della morte di Paolo Mantegazza, una sezione della quale era dedicata alle relazioni e alla corrispondenza con Charles Darwin, ci imbattemmo in questo strano corno, che disponeva di un cartellino identificativo che recitava appunto 'innesto di sperone di gallo sull'orecchio di una vacca'. Desiderosi di sapere di più riguardo a questo pezzo trovammo il lavoro di Mantegazza sugli innesti animali e il disegno di Bizzozero che senza alcuna ambiguità permise di identificare il corno come l'oggetto al centro della corrispondenza tra Mantegazza e lo scienziato inglese. Ora questo corno può raccontarci la sua affascinante storia.

\section{BIBLIOGRAFIA}

[1] Guida della prima Esposizione nazionale di storia della scienza, Firenze, Edizione dell'Ente per le Attività Toscane, 1929.

[2] Paolo Mantegazza, Degli innesti animali e della produzione artificiale delle cellule, Milano, Amministrazione del Politecnico, 1865.

[3] Paolo Mazzarello, Alberto Calligaro, Giulio Bizzozero: a pioneer of cell biology, Nature reviews molecular cell biology, 2001: 2: 776-781.

[4] Paola Govoni, Paolo Mantegazza, in Il Contributo italiano alla storia del Pensiero. Appendice VIII della Enciclopedia Italiana di Scienze, Lettere ed Arti, vol. IV, Scienze, Roma, Istituto della Enciclopedia Italiana, 2013, pp. 592-596.

[5] Paolo Mazzarello, La medicina e le discipline affini nelle pubblicazioni dell'Istituto Lombardo, in L'Istituto Lombardo - Accademia di Scienze e Lettere II - Storia delle discipline, Milano, Libri Scheiwiller, 2008, pp. 565-670.

[6] Popular science review, 1865: 4: 521-522.

[7] Janet Browne, Charles Darwin. The power of place, London, Pimlico, 2003.

[8] Charles Darwin, The variation of animals and plants under domestication, London, John Murray, 1868.

[9] Paolo Mantegazza, Studj sui matrimonj consanguinej, Rendiconti dell'Istituto Lombardo di Scienze e Lettere, 1868: 1 (s. II): 106-109.

[10] Paolo Mantegazza, Studj sui matrimonj consanguinei, Milano, Gaetano Brigola, 1868.

[11] Paolo Mantegazza, Carlo Darwin e il suo ultimo libro, Nuova Antologia, 1868: 8: 70-98.

[12] Giovanni Landucci, L'occhio e la mente. Scienze e filosofia nell'Italia del secondo Ottocento, Firenze, Oelschki, 1987. 